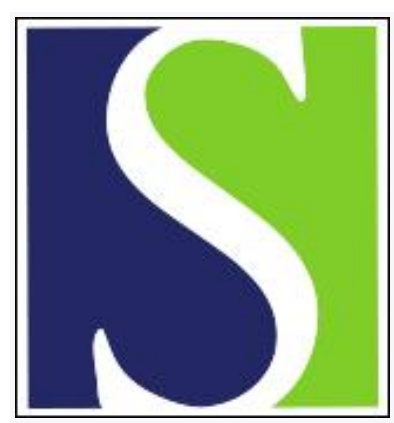

Scand J Work Environ Health 1999;25(4):305-307

https://doi.org/10.5271/sjweh.439

Issue date: Aug 1999

Do medical epidemiologists and statisticians really communicate?

by Partanen $\mathrm{T}$

This article in PubMed: www.ncbi.nlm.nih.gov/pubmed/10505655

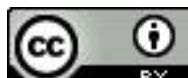


Scand J Work Environ Health 1999;25(4):305-307

\section{Do medical epidemiologists and statisticians really communicate?}

Subject matters in epidemiology have gradually become, and will yet become, increasingly complex. While some of us who were old enough back in the 1950s were asking whether tobacco smoking might increase mortality from coronary heart disease or lung cancer, epidemiologists today wonder about the complex social pathways leading to the smoking habit and its fluctuations, how to assess the feasibility and efficacy of health promotion programs in complex social settings, and how cellular macromolecules interact with doses of components of inhaled cigarette smoke and thus modify dose-response functions of the risk of histological subdivisions of lung cancer, which are further modified by genetic polymorphisms. Causation, modification, confounding, and prevention have become chained, multilevel and multidisciplinary matters. Simple risk factor epidemiology is becoming too inefficient to tackle phenomena that reach from subcellular mechanisms to macrosocial structures. At the same time, there is an countercurrent towards less formal research procedures, such as the difficulty of accepting randomization and replication in human health promotion or a striving toward data smoothing that would not "overmodel". Facing all the diversity, I would dare predict that the development of epidemiology will become all the more diversified rather than converging into some single authoritarian paradigm.

It is the medically trained person who is often in charge of an epidemiologic study. He or she often needs to lean heavily on experts in other fields, such as hygiene, dietetics, toxicology, genetics, physiology, psychology, or sociology. Statisticans, in particular, were rather early involved in epidemiologic research, with the result that epidemiologic methods have witnessed a rapid development in statistical methodologies during the past 40 years. An exception to the rule is that, in developing countries, statisticians were really not needed. Approaches were simple, and health effects were powerful enough to penetrate robust designs and analyses. Today, statistics is increasingly applied in epidemiology in the Third World. If local expertise is not available, communication is opened with "northern" collaborators (1), with concomitant problems created by the distance.

In this context, how do statisticians and medical epidemiologists understand each other, and the phenomena under study today, since both the phenomena and the statistical models have become much more complex?

Phenomena and mechanisms need to be understood as thoroughly as possible by the subjectmatter epidemiologist, usually the team leader, who then should explain them to the statistician. The statistician, after having understood and considered the subject matter and having scrutinized the data, then needs to explain his proposed statistical model to the epidemiologist. Both must understand each other. This need requires sufficient interest and devotion of time on the part of both persons. Both must also know the performance qualities (conceptual validity, empirical reproducibility, etc) of operationalizations such as the diagnostic classifications, hygienic measurements, biomarkers, and questionnaires used. The statistician certainly should be involved in the study from its very outset.

Models, especially multivariate models, are sometimes blindly applied to observational data that have "black holes" (ie, when multivariate distributions are not covered with data in crucial segments). We have recent experience in multivariate meta modeling that produced the highest meta rate ratios for nonexistent multivariate study quality combinations such as old studies using standardized mortality ratios, expert assessments of exposures, and histological confirmation of diagnoses. In order to avoid extrapolations to no-data domains, it is prudent to take time to examine multivariate distributions and to simplify analyses accordingly. I also deem it advisable to have model results checked against results 
from stratified analyses in order to keep close to the data rather than get carried away by models that may be unfit for the data. This practice also shows the subject-matter expert "hands-on" how models smooth the data and how the smoothing can sometimes lead a scientist astray.

A difficult situation arises when statisticians are heavily loaded with tightly scheduled tasks on data representing a large array of subject matters. The preferable extreme is a statistician who, in a long-term commitment or contract, is a solid part of the research team of one or a few projects in a narrow field of subject matter. The Finnish Institute of Occupational Health represents a wide field of subject matters in research, even in epidemiology alone. The bulk of the statistical and computing expertise lies at a "centralized" block of the formal organization. It was only natural that the statisticians in the block have been broadly allocated into expansive fields of subject matter such as accident prevention, physiology, psychology, occupational hygiene, genotoxicology, and occupational medicine. Even within occupational epidemiology, each of these disciplines or subject-matter fields have their counterparts and thus require at least basic substance knowledge on the part of the statistician.

Another observation is that new developments emerge all the time, both in subject matter and in methodology. Multistep and multilevel phenomena are often encountered and scrutinized. Bayesian and meta-analytic studies are here to stay. "Mechanistic" or "molecular" epidemiology brings about the need for complex interaction models and novel design requirements. In addition, small-sample statistics are back, along with biomarker studies. Costly determinations of many biomarkers of exposure and effect are reflected in small numbers of subjects. Often the data come from some unrelated primary source that dictates distributions of variables of interest. This is a particular case for the need for close communication with the statistician. Public health epidemiologists, on the other hand, learn from neighboring sciences such as the social sciences and demography. Occupational hygienists develop deterministic and stochastic models for exposure assessment. Toxicologists and pharmacologists insist on the estimation of internal doses even if there is a lack of direct data. Interview experts require the incorporation of qualitative components into the data. Epidemiology in developing countries may need an anthropological or participatory component. Each of the bordering sciences brings in its methodologies, some of which have statistical implications. Thus, for chemical agents, do we know what we are trying to estimate by intensities, durations, and cumulated external exposures? If it is the individual organ burden, do we have even a faint idea what determines the organ burden for the agent or metabolite we are studying (parameters of route, deposition, intake fractions and rates, retention, metabolic activation, clearance)? In other words, do we use hygiene data that are, to any extent, related to the unknown individual burden? Similarly, how are our operationalizations of the conceptual end points (local or systemic, acute or long-term, reversible or irreversible)? These are matters that have to become clear to the statistician and, for that matter, to the entire research team. It certainly was a delight to read about dose-exposure modeling in an occupational epidemiology textbook (2), a topic usually overlooked in such works.

Do the statistician and medical expert understand the implications of causal inference? It certainly is prudent to argue that one unique study does not usually resolve causality or establish dose-response. Do both parties then understand the problems and the solutions involved in using all available evidence? Statistical aspects are strongly implied in the incorporation of previous evidence in the causality assessment, as well as in quantitative risk assessment, which often brings in nonepidemiologic evidence and various extrapolations and their accompanying uncertainties.

How can communication and understanding be improved? My feeling is that, in the long term, academic training better not be extremely narrow for upcoming researchers in multidisciplinary teams. Second, continuous systematic training within research institutions is a must for survival. Thus, in the wake of genotoxicologic epidemiology at the Finnish Institute of Occupational Health, we have felt that genotoxicologists, medical specialists, epidemiologists, and statisticians would benefit from a joint 
exercise of reading a modern book on molecular epidemiology. [This was, at the time, the book by Hulka et al (3).] This we did chapter by chapter, one of us reading the chapter particularly carefully, then presenting the contents to the others. We then discussed the contents for understandability and from the viewpoint of potential applications at the Institute. The study circle convened 10 times during one academic semester. It seems confident to conclude that we all communicate now better with each other than we did at the outset, and we will probably have another such exercise implemented in the near future. Subsequently, a similar idea was adopted at the International Agency for Research on Cancer, and I am confident that several research institutes around the world have done something similar. The central idea is not to deliver fragmentary information now and then, but rather to go deeper into the matters on a longer term systematic basis.

Curriculums and PhD programs in medicine, epidemiology, public health, and biostatistics have probably improved over the last few decades to enhance the understanding of subject matter and statistics, with the result that graduates are, on the average, more learned and hopefully more multidisciplinary than in the 1950s. Yet, leaders of research institutes and departments better make sure to have their staff constantly trained in order not to fall behind during times when knowledge is increasing at a rate thus far never experienced. For this purpose time for study circles, reading, and discussion is needed. It also requires open-mindedness, motivation, and mutual trust between scientists with different backgrounds.

\section{References}

1. Partanen TJ, Hogstedt C, Ahasan R, Aragón A, Arroyave ME, Jeyaratnam J, et al. Collaboration between developing and developed countries and between developing countries in occupational health research and surveillance. Scand J Work Environ Health 1999;25(3):296-300.

2. Checkoway H, Pearce NE, Crawford-Brown DJ. Research methods in occupational epidemiology. Oxford: Oxford University Press, 1989.

3. Hulka BS, Wilcosky TC, Griffith ID. Biological markers in epidemiology. Oxford: Oxford University Press, 1990.

Timo Partanen, $P h D$

Department of Epidemiology and Biostatistics

Finnish Institute of Occupational Health

Topeliuksenkatu $41 \mathrm{~A}$

FIN-00250 Helsinki Finland

[timo.partanen@occuphealth.fi] 